\title{
Prevalência de hipertensão arterial em pacientes com oclusão do ramo da veia central da retina
}

\author{
Prevalence of arterial hypertension in branch retinal vein occlusion patients
}

\author{
Alexandre Antonio Marques Rosa ${ }^{1}$ \\ Kátia Coelho Ortega' ${ }^{2}$ \\ Décio Mion Jr. ${ }^{3}$ \\ Yoshitaka Nakashima ${ }^{4}$
}

\begin{tabular}{|c|}
\hline RESUMO \\
\hline 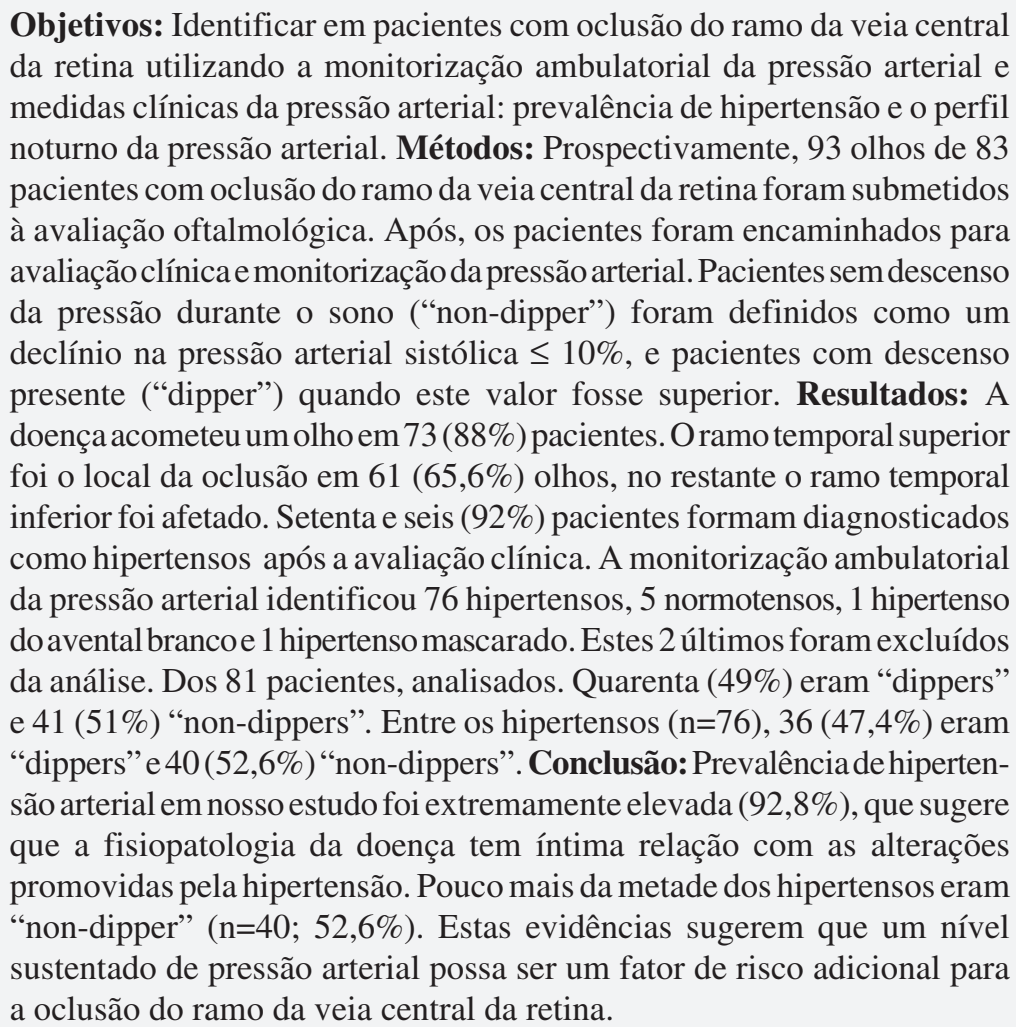 \\
\hline
\end{tabular}

Descritores: Hipertensão/diagnóstico; Pressão arterial; Oclusão da veia retiniana; Veia retiniana/patologia; Doenças retinianas/fisiopatologia

\section{INTRODUÇ̃̃̃O}

As oclusões venosas são a segunda causa mais comum de doenças vasculares da retina, atrás apenas da retinopatia diabética ${ }^{(1-2)}$, sendo o motivo mais comum de perda visual súbita em pacientes hipertensos ${ }^{(3)}$.

A oclusão do ramo da veia central da retina (ORVCR) é definida como uma oclusão focal de uma veia retiniana ao nível de um cruzamento arteriovenoso (AV), onde a artéria passa anteriormente à veia. Neste local, artéria e veia apresentam uma bainha adventícia comum ${ }^{(4)}$.

A hipertensão arterial é uma doença multifatorial, conceituada como síndrome, caracterizada pela presença de níveis tensionais elevados, associados às alterações metabólicas e hormonais e a fenômenos tróficos (hipertrofias cardíaca e vascular) ${ }^{(5)}$. 
O aumento da pressão arterial grave, tanto essencial como secundária a outras doenças sistêmicas, tem um amplo efeito sobre os vasos sanguíneos de todo o organismo ${ }^{(5)}$.

A hipertensão é um fator de risco particularmente importante no desenvolvimento das ORVCR ${ }^{(6-13)}$, sendo alta sua prevalência entre estes pacientes. Contudo, a definição de hipertensão nos trabalhos que avaliam os fatores de risco para o desenvolvimento de ORVCR nem sempre é muito clara ou não obedece às normas técnicas exigidas ${ }^{(5)}$.

A monitorização ambulatorial da pressão arterial (MAPA) é melhor que a medida ambulatorial isolada na detecção de lesões em órgão alvo ${ }^{(14-15)}$. Este é o primeiro estudo que segue rigorosamente as normas das Diretrizes Brasileiras de Hipertensão Arterial para aferição da pressão arterial no consultório, e na avaliação do perfil de comportamento da pressão nas 24 horas por meio da MAPA em pacientes com ORVCR.

O objetivo do presente estudo foi determinar a prevalência de hipertensos entre os pacientes com ORVCR, utilizando as medidas clínicas ambulatoriais, bem como a MAPA, com o intuito de identificar casos de hipertensão mascarada (normotensão do avental branco).

\section{MÉTODOS}

O protocolo de pesquisa, bem como o Termo de Consentimento Livre e Esclarecido, foi aprovado pela Comissão de Ética do Hospital de Clínicas da Faculdade de Medicina da Universidade de São Paulo (HCFMUSP).

Foram avaliados pacientes de ambos os sexos, com idade superior a 21 anos e com suspeita ou diagnóstico prévio de ORVCR. Todos os pacientes foram examinados pelo mesmo médico (AAMR).

Foram excluídos pacientes com outros tipos de oclusão venosa da retina, que não a de ramo; portadores de hemoglobinopatias e/ou hiperviscosidade sanguínea (hipergamaglobulinemia, macroglobulinemia de Waldenström's, policitemia e leucemias); histórico de uveíte (intermediária ou posterior) recente; casos de oclusão de ramo venoso onde não foi possível identificar o cruzamento AV acometido e histórico de traumatismo ocular recente.

A consulta oftalmológica foi realizada na Clínica Oftalmológica do HCFMUSP, consistiu de medida da acuidade visual com e sem correção, refratometria, biomicroscopia, tonometria de aplanação e exame de fundo de olho, para confirmação diagnóstica.

As oclusões foram classificadas como sendo do ramo venoso temporal ou nasal após a localização do cruzamento AV acometido. Foram subdivididas em ramo superior ou inferior de acordo com sua posição na retina.

Os pacientes foram divididos em fase aguda ou crônica segundo o tempo de início dos sintomas, menor ou igual há seis meses ou maior que seis meses, respectivamente. Pacientes com oclusões em ambos os olhos, com intervalo de acometimento superior a seis meses, foi considerado para efeito da determinação da fase (aguda ou crônica) o olho mais recentemente acometido.

Após avaliação oftalmológica, os pacientes foram encaminhados para Unidade de Hipertensão da Disciplina de Nefrologia do HCFMUSP para avaliação clínica, realização de exames laboratoriais e da MAPA.

A medida da pressão arterial foi realizada de acordo com as recomendações da Sociedade Brasileira de Hipertensão(5). Um método indireto com técnica auscultatória utilizando um esfigmomanômetro de coluna de mércurio calibrado (Baummometer, Desk Model, New York, EUA) foi empregado. Os pacientes permaneceram sentados e em repouso por pelo menos 10 minutos antes das medidas. Foram orientados a não comer, não ingerir bebidas com álcool ou cafeína e não fumar pelo menos 30 minutos antes dos exames. Além disso, foram questionados quanto à vontade de ir ao banheiro antes das medidas. As medidas da pressão arterial foram tomadas utilizando manguito de tamanho adequado de acordo com a circunferência do braço do paciente (manguito de $13 \times 22 \mathrm{~cm}$ para membros superiores com circunferência de 26 a $32 \mathrm{~cm}$ e manguito de 17 x $32 \mathrm{~cm}$ para membros superiores com circunferência de $33 \mathrm{a} 42 \mathrm{~cm}$ ).

Foram realizadas 5 medidas ambulatoriais da pressão arterial, e a média das duas últimas foi utilizada para definir a presença ou não de hipertensão. Se após 5 medidas, a diferença das duas últimas fosse superior a $4 \mathrm{mmHg}$, as medidas foram repetidas até que houvesse diferença menor do que $4 \mathrm{mmHg}$.

Paciente hipertenso foi definido como aquele em uso de medicação anti-hipertensiva e/ou com medida de pressão sistólica maior ou igual a $140 \mathrm{mmHg}$ ou pressão diastólica maior ou igual a $90 \mathrm{mmHg}^{(5)}$.

Os hipertensos foram classificados quanto ao estágio da hipertensão (leve, moderada e grave) de acordo com os critérios da diretrizes brasileira de hipertensão ${ }^{(5)}$.

A MAPA foi realizada com equipamento oscilométrico validado e calibrado (SpaceLabs 90207, SpaceLabs Inc, Richmond, WA, USA). O manguito adequado para a circunferência do braço foi colocado no braço não dominante e o monitor foi colocado na cintura com um cinto. Os pacientes foram instruídos a manter o braço estendido ao longo do corpo e não movêlo durante as medidas. O monitor foi programado para obter medidas a cada 15 minutos das $06 \mathrm{~h} 01$ às $22 \mathrm{~h} 00 \mathrm{e}$ a cada 20 minutos das $22 \mathrm{~h} 01$ às $06 \mathrm{~h} 00$.

Cada paciente foi orientado a anotar as atividades realizadas durante o dia, bem como o horário em que foi deitar e o horário que despertou. Estas anotações foram utilizadas para a definição do período real de sono e de vigília.

Foram adotados os valores de normalidade da MAPA recomendados pela Sociedade Brasileira de Hipertensão ${ }^{(16)}$.

Pela MAPA foi identificada a ocorrência de: a) hipertensão do avental branco - caracterizada por valores anormais na medida da pressão arterial no consultório (igual ou acima de $140 / 90 \mathrm{mmHg}$ ) e comportamento normal pela MAPA de vigília (igual ou abaixo de 135/85 mmHg); e b) normotensão do avental branco ou hipertensão mascarada - valores normais na medida da pressão arterial 
no consultório (abaixo de 140/90 mmHg) e valores anormais pela MAPA durante o período de vigília (acima de $135 / 85 \mathrm{mmHg})^{(16)}$. Pacientes com hipertensão ou normotensão do avental branco foram excluídos da análise.

Foi definida como presença de descenso da pressão durante o sono uma diminuição da pressão arterial sistólica (PAS) na MAPA $>10 \%$ e ausência de descenso quando este valor fosse inferior $(\mathrm{PAS} \leq 10 \%)$.

\section{RESULTADOS}

Para o processamento e análise estatística, foi construído um banco de dados utilizando programa específico (Statistical Package for the Social Sciences - SPSS for Windows ${ }^{\circledR}$, v 11.0).

As variáveis qualitativas foram representadas por frequiência absoluta (n) e relativa (\%) e as quantitativas pela média, desvio padrão, valores mínimo e máximo. Em todos os testes estatísticos adotou-se um nível de significância de $0,05(\alpha=5 \%)$.

Foram examinados, prospectivamente, 107 pacientes no período de janeiro de 2003 a março de 2004. Nesta etapa, 18 pacientes foram excluídos por não apresentarem ORVCR e seis, devido impossibilidade de identificar o cruzamento AV acometido por algum tipo de opacidade de meios (catarata ou hemorragia vítrea). A população do estudo ficou composta inicialmente por 83 pacientes (93 olhos) com oclusões venosas de ramo.

Havia $48(57,8 \%)$ pacientes do sexo feminino, com idades entre 42 e 79 anos, média de 59,4 $\pm 10,0$ anos. A doença manifestou-se em apenas um dos olhos em $73(88 \%)$ pacientes.

Em todos os casos, foi identificado qual(is) o(s) cruzamento(s) arteriovenoso(s) acometido(s). A artéria estava anterior à veia em todos (100\%) os casos. Em $61(65,6 \%)$ olhos, o ramo venoso retiniano acometido foi o da arcada temporal superior. Não foi diagnosticado nenhum caso acometendo vasos do setor nasal.

A média de início dos sintomas foi 21,0 $\pm 27,8$ meses. Dos 83 pacientes, $39(46,9 \%)$ casos corresponderam a obstruções venosas agudas ( $\leq 6$ meses), cuja média de início da doença foi $2,7 \pm 1,9$ meses. Houve $44(53,1 \%)$ casos crônicos (> 6 meses), com média de início dos sintomas de 36,4 $\pm 31,8$ meses.

Entre os pacientes com oclusão venosa, não foram encontradas alterações hematológicas. Quinze pacientes $(18,1 \%)$ apresentavam glicemia superior a $110 \mathrm{mg} / \mathrm{dl}$ ou hemoglobina glicada superior $7 \%$. Além destes, mais três pacientes $(3,6 \%)$, cujos níveis foram inferiores, faziam uso regular de medicação para diabete. Quarenta pacientes $(48,2 \%)$ apresentavam valores de colesterol total superior a $200 \mathrm{mg} / \mathrm{dl}$ ou de colesterol LDL ("Low density lipoprotein") inferior a $140 \mathrm{mg} / \mathrm{dl}$.

Dezesseis indivíduos $(19,2 \%)$ apresentavam pressão intraocular superior a $21 \mathrm{mmHg}$. Dentre estes, apenas $4(25 \%)$ já faziam uso, de maneira irregular, de medicação para redução da pressão intra-ocular.

Após a avaliação clínica, foram identificados 76 (92\%) hipertensos e 7 (8\%) normotensos. A MAPA identificou 76 hipertensos, 5 normotensos, 1 hipertensão do avental branco e 1 normotensão do avental branco. Estes 2 últimos foram excluídos da análise, portanto, 81 pacientes foram analisados para determinação do comportamento da pressão arterial durante o sono em indivíduos com ORVCR.

Dos 81 pacientes, 40 (49\%) apresentaram descenso da pressão arterial sistólica durante o sono ("dippers") enquanto que 41 (51\%) não apresentaram ("non-dippers").

Avaliando-se apenas os hipertensos $(\mathrm{n}=76), 36(47,4 \%)$ apresentaram descenso da pressão arterial sistólica durante o sono enquanto que $40(52,6 \%)$ não apresentaram. Entre os normotensos ( $n=5)$, apenas um paciente (20\%) não apresentava descenso da pressão durante o sono.

\section{DISCUSS ÃO}

Na maioria dos olhos ( $\mathrm{n}=61 ; 65,6 \%)$, o ramo venoso temporal superior foi o local da obstrução. Não foi observado nenhum caso acometendo vasos do setor nasal. Talvez por tais casos causarem pouca sintomatologia, o seu diagnóstico seja menos comum. Este dado é semelhante ao observado por outros autores $^{(17)}$, onde há maior prevalência de oclusões no setor temporal, sobretudo acometendo o ramo temporal superior.

As possíveis explicações para a maior freqüência de lesões do ramo temporal superior seriam: (1) Percepção mais precoce da doença devido diminuição da acuidade visual por envolvimento da mácula e (2) maior número de cruzamentos arteriovenosos no setor temporal superior ${ }^{(17)}$.

A ORVCR afeta indivíduos de ambos os sexos, sendo mais freqüente entre 60 a 70 anos de idade ${ }^{(8)}$. Neste trabalho, a média de idade dos pacientes foi 59,4 $\pm 10,0$ anos.

Como já descrito na litetratura ${ }^{(6)}$, foi observado neste estudo maior prevalência (88\%) de casos unilaterais. Apenas 10 (12\%) pacientes apresentaram o acometimento de ambos os olhos, de forma não simultânea.

O diagnóstico da hipertensão arterial é estabelecido pelo encontro de níveis tensionais acima dos limites de normalidade (pressão sistólica maior ou igual que $140 \mathrm{mmHg}$ ou pressão diastólica maior ou igual a $90 \mathrm{mmHg})^{(5)}$.

A pressão sanguínea elevada é uma condição comum que não têm manifestações clínicas específicas até o desenvolvimento de lesões em órgãos alvo ${ }^{(18)}$.

Uma aferição adequada é de fundamental importância ${ }^{(18)}$. As causas mais comuns de falhas na aferição da pressão arterial são: manguito de tamanho inadequado (97\%), não permitir um repouso mínimo antes de iniciar as medidas (96\%), deflação muito rápida ( $82 \%)$, não verificação da pressão nos dois braços $(77 \%)$ e incapacidade de palpar a pressão sistólica máxima antes da ausculta $(62 \%)^{(19)}$.

A hipertensão arterial sistêmica é um fator de risco particularmente importante para as oclusões venosas de ramo, sua prevalência varia de 53 a $74 \%^{(6-13)}$. Nestes trabalhos, a definição de hipertensão arterial nem sempre é a mesma ou não obedece às normas técnicas exigidas ${ }^{(5)}$. 
Em alguns estudos ${ }^{(7,11)}$, apenas a história pregressa de hipertensão ou o uso de medicação anti-hipertensiva é levada em conta para classificar um indivíduo como hipertenso. Em outros, apenas medidas ambulatoriais da pressão foram realizadas, e os valores para considerar um indivíduo hipertenso variam de $140 / 90$ a $160 / 95 \mathrm{mmHg}^{(6,8-10,12-13)}$.

A monitorização ambulatorial da pressão arterial (MAPA) é um método automático de medida indireta e intermitente da pressão arterial durante 24 horas, enquanto o paciente realiza suas atividades rotineiras, inclusive durante o sono.

As evidências relacionadas à lesão em órgãos alvo causadas pela hipertensão arterial sugerem que a monitorização ambulatorial da pressão arterial (MAPA) está mais intimamente relacionada com este tipo de lesão ${ }^{(14-15)}$.

Uma possível explicação seria que as medidas obtidas no consultório médico não refletem a carga pressórica global imposta sobre o coração e o sistema arterial devido a amplas variações que ocorrem durante as atividades cotidianas ${ }^{(20)}$.

A pressão arterial tem características próprias nas 24 horas, apresentando maiores valores no período de vigília e queda fisiológica durante o sono ${ }^{(21)}$.

Há situações, entretanto, nas quais pode haver ausência, atenuação ou até inversão deste descenso fisiológico da pressão arterial. Indivíduos com esta característica são chamados de "non-dippers" sendo distinguidos daqueles com comportamento fisiológico, chamados de "dippers"(22).

A prevalência de indivíduos "non-dippers" varia entre os diversos estudos de $17,1 \%{ }^{(22)}$ a $40 \%{ }^{(23)}$. Estas evidências sugerem que um nível mais sustentado de pressão arterial nas 24 horas possa ser um fator de risco importante para o desenvolvimento de oclusões venosas.

Vários estudos demonstraram a importância prognóstica da falta de queda da pressão arterial durante o sono, correlacionando este fato com uma maior incidência de lesões em órgãosalvo $^{(23-24)}$. De maneira semelhante, pacientes com oclusões venosas também têm maior risco de eventos cardiovasculares ${ }^{(25-27)}$.

A preponderância das mortes relacionadas à hipertensão ocorre na faixa limítrofe hipertenso-normotenso, como resultado de um grande número de pessoas com estes níveis de pressão $\operatorname{arterial}^{(28)}$.

Portanto, medidas baixas ou normais da pressão arterial no consultório podem excluir do diagnóstico de hipertensão nestes indivíduos com normotensão do avental branco, expondo-os às conseqüências a longo prazo da doença.

Nossa hipótese era que a MAPA pudesse identificar casos de hipertensão mascarada entre os indivíduos normotensos, e que entre os hipertensos houvesse uma maior prevalência de "non-dippers".

Faz sentido se pensar que um nível mais sustentado de pressão possa predispor ao aparecimento de oclusões venosas de ramo, devido às alterações induzidas na parede arterial e conseqüente alteração ao nível do cruzamento AV.

Contudo, a prevalência de hipertensão arterial no estudo atual foi extremamente alta (92\%), após as medidas ambulato- riais. Os valores da MAPA foram muito semelhantes $(93,8 \%)$, identificando apenas um caso de paciente normotenso e um caso de hipertensão do avental branco. Isto sugere que a fisiopatologia da doença está intimamente relacionada a alterações vasculares causadas pelo aumento da pressão arterial.

Pouco mais da metade dos pacientes não apresentava descenso da pressão arterial durante o sono $(n=41 ; 51 \%)$. Se for considerada apenas os hipertensos, foi obtida uma prevalência de "non-dippers" semelhante $(\mathrm{n}=40 ; 52,6 \%)$.

Talvez a falta de queda da pressão durante o sono seja um fator importante ou adicional, pois aparece em metade dos pacientes, mas não fundamental na gênese da doença.

Estudos descritivos são normalmente seguidos de estudos analíticos, para descobrir possível relação de causa-efeito. Posteriormente, seria interessante obter um grupo controle de pacientes hipertensos sem obstrução venosa que permitiria desenvolver um estudo tipo caso-controle para calcular o risco relativo das variáveis estudadas neste trabalho.

\section{CONCLUSÕES}

A prevalência da hipertensão arterial nos portadores de ORVCR, neste estudo original, foi extremamente alta, tanto após as medidas clínicas ambulatorias como na MAPA, sugerindo que a fisiopatologia desta doença está intimamente relacionada a alterações vasculares causadas pelo aumento da pressão arterial.

Pouco mais da metade dos pacientes não apresentava descenso da pressão arterial durante o sono. Talvez a falta de queda da pressão durante o sono seja um fator importante ou adicional, mas não fundamental na gênese da doença, devido a maior sobrecarga imposta aos vasos sanguíneos durante as 24 horas.

\section{ABSTRACT}

Purposes: To identify in patients with branch retinal vein occlusion using ambulatory blood pressure monitoring and clinical blood pressure measures: hypertension prevalence, and nocturnal profile of blood pressure. Methods: Prospectively, 93 eyes of 83 patients with branch retinal vein occlusion were submitted to ophthalmological examination. Afterwards the patients were submitted to clinical evaluation and blood pressure monitoring. Non-dipper was defined as a fall in systolic blood pressure $\leq 10 \%$, and dipper when this value was higher. Results: Disease affected one eye in 73 (88\%) patients. The temporal superior branch was the site of occlusion in 61 $(65.6 \%)$ eyes, while in the others the infero-temporal branch was affected. Seventy six (92\%) patients were diagnosed as hypertensive after clinical evaluation. Ambulatory blood pressure monitoring identified 76 hipertensives, 5 normotensives, 1 white-coat hypertensive and one masked hypertensive subjects. The two latter were excluded from the analysis. Of the 81 analyzed patients, forty (49\%) were dippers and 41 (51\%) 
were non-dippers. Among the HT ( $\mathrm{n}=76), 36$ (47\%) were dippers and 40 (53\%) were non-dippers. Conclusion: Prevalence of hypertension in our series was extremely high $(92 \%)$ which suggests that physiopathology of the disease has a close relationship with changes promoted by hypertension. A little more than half of the hypertensives were non-dippers $(n=40 ; 52,6 \%)$. These evidences suggest that a 24-hour sustained level of blood pressure may be an additional risk factor for branch retinal vein occlusion.

Keywords: Hypertension/diagnosis; Blood pressure; Retinal vein occlusion; Retinal vein; Retinal diseases/physiopathology

\section{REFERÊNCIAS}

1. Argon laser photocoagulation for macular edema in branch vein occlusion. Branch Vein Occlusion Study Group Am J Ophthalmol. 1984;98(3):271-82.

2. Rosa AA. Oclusão de ramo da veia central da retina. Arq Bras Oftalmol. 2003; 66(6):897-900.

3. Weinberg D, Dodwell DG, Fern SA. Anatomy of arteriovenous crossings in branch retinal vein occlusion. Am J Ophthalmol. 1990;109(3):298-302. Comment in: Am J Ophthalmol. 1990;110(1):97-8.

4. Staurenghi G, Lonati C, Aschero M, Orzalesi N. Arteriovenous crossing as a risk factor in branch retinal vein occlusion. Am J Ophthalmol. 1994;117(2):211-3 Comment in: Am J Ophthalmol. 1994;118(2):263-5.

5. Sociedade Brasileira de Hipertensão; Sociedade Brasileira de Cardiologia; Sociedade Brasileira de Nefrologia. IV Diretrizes Brasileiras de Hipertensão Arterial. Arq Bras Cardiol. 2004;82(Supl 4):7-22.

6. Johnston RL, Brucker AJ, Steinmann W, Hoffman ME, Holmes JH. Risk factors of branch retinal vein occlusion. Arch Ophthalmol. 1985;103(12):1831-2.

7. Rath EZ, Frank RN, Shin DH, Kim C. Risk factors for retinal vein occlusions A case-control study. Ophthalmology. 1992;99(4):509-14. Comment in: Ophthalmology. 1992;99(12):1754-6.

8. Risk factors for branch retinal vein occlusion. Eye Disease Case-Control Study Group Am J Ophthalmol. 1993;116(3):286-96

9. Glacet-Bernard A, Coscas G, Chabanel A, Zourdani A, Lelong F, Samama MM Prognostic factors for retinal vein occlusion: prospective study of 175 cases. Ophthalmology. 1996;103(4):551-60.

10. Mitchell P, Smith W, Chang A. Prevalence and associations of retinal vein occlusion in Australia. The Blue Mountains Eye Study. Arch Ophthalmol. 1996;114(10):1243-7.

11. Simons BD, Brucker AJ. Branch retinal vein occlusion. Axial length and other risk factors. Retina. 1997;17(3):191-5.

12. Hayreh SS, Zimmerman B, McCarthy MJ, Podhajsky P. Systemic diseases associated with various types of retinal vein occlusion. Am J Ophthalmol. 2001; 131(1):61-77.
13. Beaumont PE, Kang HK. Clinical characteristics of retinal venous occlusions occurring at different sites. Br J Ophthalmol. 2002;86(5):572-80.

14. Mancia G, Zanchetti A, Agabiti-Rosei E, Benemio G, De Cesaris R, Fogari R, et al. Ambulatory blood pressure is superior to clinic blood pressure in predicting treatment-induced regression of left ventricular hypertrophy. SAMPLE Study Group on Ambulatory Monitoring of Blood Pressure and Lisinopril Evaluation. Circulation. 1997;95(6):1464-70. Erratum in: Circulation. 1997;96(3):1065. Agebiti-Rosei E [corrected to Agabiti-E]; Pessino A [corrected to Pessina A].

15. Parati G, Pomidossi G, Albini F, Malaspina D, Mancia G. Relationship of $24 \mathrm{~h}$ blood pressure mean and variability to severity of target-organ damage in hypertension. J Hypertens. 1987;5(1):93-8.

16. Sociedade Brasileira de Hipertensão. III Diretrizes para uso da Monitorização Ambulatorial da Pressão Arterial. I Diretrizes para uso da monitorização residencial da pressão arterial III MAPA/IMRPA. Hipertensão [periódico na Internet]; 2001;4(1):6-22. [citado 2007 Jun 21]. Disponível em: http:// www.sbh.org.br/documentos/mapa_mrpa3.pdf

17. Zhao J, Sastry SM, Sperduto RD, Chew EY, Remaley NA. Arteriovenous crossing patterns in branch retinal vein occlusion. The Eye Disease CaseControl Study Group. Ophthalmology. 1993;100(3):423-8.

18. McAlister FA, Straus SE. Evidence based treatment of hypertension. Measurement of blood pressure: an evidence based review. BMJ. 2001;322(7291):908-11. Comment in: BMJ. 2001;323(7309):399-400.

19. McKay DW, Campbell NRC, Parab LS, Chockalingam A, Fodor JG. Clinical assessment of blood pressure. J Hum Hypertens. 1990;4(6):639-45.

20. Liu JE, Roman MJ, Pini R, Schwartz JE, Pickering TG, Devereux RB. Cardiac and arterial target organ damage in adults with elevated ambulatory and normal office blood pressure. Ann Intern Med. 1999;131(8): 564-72. Comment in: Ann Intern Med. 2000;132(10):842.

21. Millar-Craig MW, Bishop CN, Raftery EB. Circadian variation of bloodpressure. Lancet. 1978;1(8068):795-7.

22. O'Brien E, Scheridan J, O'Malley K. Dippers and non-dippers [letter]. Lancet. 1988;2(8607):397.

23. Verdecchia P, Schillaci G, Guerrieri M, Gatteschi C, Benemio G, Boldrini F, Porcellati C. Circadian blood pressure changes and left ventricular hypertrophy in essential hypertension. Circulation. 1990;81(2):528-36. Comment in: Circulation. 1990;81(2):700-2.

24. Verdecchia P, Porcellati C, Schillaci G, Borgioni C, Ciucci A, Battistelli M, et al. Ambulatory blood pressure. An independent predictor of prognosis in essential hypertension. Hypertension. 1994;24(6):793-801. Erratum in: Hypertension. 1995; 25(3):462. Comment in: Hypertension. 1995;25(5):1116-7.

25. Martin SC, Butcher A, Martin N, Farmer J, Dobson PM, Bartlett WA, et al Cardiovascular risk assessment in patients with retinal vein occlusion. $\mathrm{Br} \mathrm{J}$ Ophthalmol. 2002;86(7):774-6. Comment in: Br J Ophthalmol. 2003;87(1):124.

26. Wong TY, Larsen EK, Klein R, Mitchell P, Couper DJ, Klein BE, et al. Cardiovascular risk factors for retinal vein occlusion and arteriolar emboli: the Atherosclerosis Risk in Communities \& Cardiovascular Health studies. Ophthalmology. 2005;112(4):540-7.

27. Tsaloumas MD, Kirwan J, Vinall H, O'Leary MB, Prior P, Kritzinger EE, et al. Nine year follow-up study of morbidity and mortality in retinal vein occlusion. Eye. 2000;14(Pt 6):821-7.

28. Pickering TG, Devereux RB. Ambulatory monitoring of blood pressure as a predictor of cardiovascular risk. Am Heart J. 1987;114(4 Pt 2):925-8. 